\title{
Serum Triglycerides Fasting Glycemic Product Index Predict No-Reflow Phenomenon in Patients with Actue Myocardial Infarction Undergoing Primary Coronary Intervention
}

\section{LiPing Liao ( $\sim$ Callous1027@163.com )}

Shanghai No 1 Renmin Hospital: Shanghai General Hospital

\section{Yang Yang}

First Affiliated Hospital of Anhui Medical University

Zi Xuan Ye

First Affiliated Hospital of Anhui Medical University

\section{Zheng Du}

First Affiliated Hospital of Anhui Medical University

Weizhen Li

Shanghai General Hospital

\section{Xiajun Shi}

Tongling People's Hospital

\section{Research Article}

Keywords: Acute myocardial infarction, Percutaneous coronary intervention, No reflow, myocardial contrast echocardiography, CSI, WMSI, TyG index

Posted Date: November 15th, 2021

DOI: https://doi.org/10.21203/rs.3.rs-978279/v1

License: (c) (i) This work is licensed under a Creative Commons Attribution 4.0 International License. Read Full License 


\section{Abstract}

Objective: To investigate the value of serum triglyceride and fasting glycemic index in predicting no reflow in patients with acute myocardial infarction after percutaneous coronary intervention. Methods: A total of 1037 patients with acute myocardial infarction who received $\mathrm{PCl}$ in the Department of Cardiology of Shanghai General Hospital and Jiading Branch of Shanghai General Hospital from January 2016 to May 2021 were retrospectively selected. According to postoperative TIMI blood flow classification, all patients were divided into no reflow group (TIMI blood flow $\leq$ grade 2) (309 cases) and reflow group (TIMI blood flow $=$ grade 3) (728 cases). Clinical data, laboratory indicators and surgical information were collected from the two groups of patients. Logistic univariate and multivariate regression were used to analyze the independent risk factors of no reflow in actue myocardial infarction patients after PCl. ROC curve was used to analyze the best cut-off point for TyG index to predict the occurrence of no reflow.

Results: The TyG index of no reflow group was significantly higher than of reflow group $(7.16 \pm 0.64 \mathrm{vs}$ $6.63 \pm 0.38, P=0.001)$. Multivariate logistic regression analysis showed that TyG index $(O R=1.484,95 \% \mathrm{Cl}$ 1.203-1.831, $\mathrm{P}<0.001)$;WMSI(OR=2.640,95\% Cl1.036-4.722,P=0.039);CSI (OR=2.299,95\%Cl1.117-

$4.767, \mathrm{P}=0.022$ ) was independent predictors of no reflow phenomenon in patients with acute myocardial infarction after $\mathrm{PCl}$. The area under the ROC curve predicted by TyG index after $\mathrm{PCl}$ without reflow was $0.809,95 \% \mathrm{Cl}(0.740-0.879)$, higher than $\mathrm{CSI}$ and WMSI.When the TyG index was 6.995 , the sensitivity and specificity of Youden index were $82.4 \%$ and $70.3 \%$ respectively at the maximum.

Conclusion: TyG index can be used as an indicator to predict the occurrence of no-reflow in patients with acute myocardial infarction after $\mathrm{PCl}$, which is helpful for clinicians to select high-risk patients and take interventions timely, so as to reduce the risk of no-reflow in patients after $\mathrm{PCl}$.

\section{Introduction}

The pathophyseologic mechanism of acute myocardial infarction is rupture and erosion of unstable plaque of coronary artery and sharp reduction or interruption of coronary blood supply[1].Myocardial inadequacy of blood perfusion, acute ischemic necrosis occurs. The disease has the characteristics of urgent onset and high fatality rate, which is a serious threat to human life and health. Percutaneous coronary intervention (PCl) has become one of the important treatments of patients with acute myocardial infarction (AMI), which can open the occluded coronary arteries in time, save the ischemic heart muscle and improve the survival rate of patients. In clinical practice, we found that some patients suffered from acute reperfusion injury due to $\mathrm{PCl}$ surgery, leading to poor prognosis. As a serious complication of $\mathrm{PCl}$ surgery, "no reflow" phenomenon has been proved to be associated with poor prognosis in patients[2]. At present, the commonly used method used to evaluate the myocardial perfusion for myocardial acoustic imaging(MCE). However,the examination is expensive and not suitable for all people[3].Therefore, it is of great clinical significance to find a simple indicator that can be used to predict the occurrence of "no reflow"in patients with myocardial infarction after PCl. 
At present, the occurrence mechanism of "no reflow "phenomenon has not been clarified. Some studies have shown that it is associated with coronary artery spasm and contraction[4], atherosclerotic plaque embolization[5], and severe glucose metabolism disorders[6]. TyG index has been proved to be a novel marker of insulin resistance[7], and is also associated with coronary atherosclerosis[8], glucose and lipid metabolism disorders[9-10], and microvascular endothelial dysfunction[11]. The above factors are also important pathological mechanisms for the occurrence of "no reflow "phenomenon after PCl. At present, most of the studies on TyG index and cardiovascular diseases focus on the analysis of prognostic value, and there is no research report on the relationship between TyG index and "no reflow" phenomenon after $\mathrm{PCl}$. The purpose of this study was to explore the relationship between TyG index in acute myocardial patients and the absence of coronary reflow after $\mathrm{PCl}$, in order to provide clinical reference for the prevention of the absence of reflow.

\section{Objects And Methods}

\subsection{Research Objects}

This retrospective study included 1037 patients with acute myocardial infarction were hospitalized and received PCl treatment in the Department of Cardiology of Shanghai General Hospital and Jiading Branch of Shanghai General Hospital from January 2016 to May 2021, including 573 males and 464 females, aged $(64.84 \pm 13.68)$ years. All enrolled patients were divided into two groups according to TIMI blood grade after PCl. Among them, 309 patients in no-reflow group, including 181 males and 128 females, aged (65.76 \pm 12.68$)$ years; 728 patients in reflow group, including 406 males and 322 females, aged $(62.89 \pm 13.54)$ years.

\section{Exclusion criteria:}

$\triangle$ Patients with congenital heart disease, valvular heart disease, rheumatic heart disease, cardiomyopathy and pulmonary heart disease; $₫$ Patients who had undergone $\mathrm{PCl}$ or coronary artery bypass grafting, had the history of old myocardial infarction or decompensated heart failure; $₫$ Patients with confirmed familial hypertriglyceridemia; (4) Patients who had taken statins and triglyceride lowering drugs before admission;

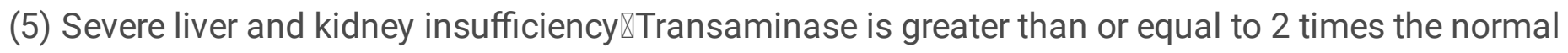

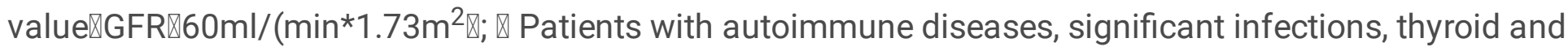
adrenal dysfunction, blood diseases, malignant diseases, and a history of surgical trauma 3 months before admission. $\otimes$ Patients with fasting glucose level higher than $6.9 \mathrm{mmol} / \mathrm{L}$ or taking hypoglycemic drugs.All study protocols of participants were reviewed and approved by The Medical Ethics Committe of JiaDing branch of Shanghai General hospital and followed the recommendations of Declaration of Helsinki.

\subsection{Diagnostic criteria and definition}

Acute myocardial infarction: Chest pain with new ST-segment changes and elevated markers of myocardial necrosis to at least twice the upper normal value[12]. TIMI blood grade criteria: Grade 0: No 
forward blood flow through infarct-related vessels; Grade 1: The contrast agent partially passes through the diseased vessels, and the distal vascular bed cannot be completely filled by the contrast agent; Level 2: contrast agent can fill the entire vascular bed, but the filling and emptyspeed is very slow, more than 3 cardiac cycles; Level 3: The contrast agent rapidly fills the distal vascular bed and empties it rapidly over 3 cardiac cycles. No reflow: postoperative TIMI blood flow $\leq 2$ (Figure 1). Reflow was defined as TIMI flow 3.(Figure 2)

\subsection{Research Methods:}

1.3.1 General data: General clinical data such as gender, age, height, weight, hypertension and smoking history of the patients were collected through the electronic medical record system.

1.3.2 Laboratory data: after admission, $3 \mathrm{ml}$ fasting elbow venous blood was collected from all patients and placed in anticoagulant tubes for examination. Roche Cobas8000 automatic biochemical analyzer was used to detect the blood biochemical indexes of patients, and SYSMEX XN-9000 automatic blood cell analyzer was used to detect and analyze myocardial injury markers, blood routine and other items. TyG index $=\mathrm{Ln}[$ serum triglyceride $(\mathrm{mg} / \mathrm{dl}) \times$ fasting glucose $(\mathrm{mg} / \mathrm{dl}) / 2]$. All patients received oral aspirin $300 \mathrm{mg}$, ticagrelor $180 \mathrm{mg}$, and low molecular weight heparin $4100 \mathrm{IU}$ intravenously before surgery. Innova 3100 fully digital angiography machine was used, with iodipin as contrast agent. PCl operation and TIMI blood grade diagnosis were performed by 2 or more experienced senior physicians with intervention qualification.

\subsubsection{Echocardiography and myocardial contrast echocardiography;}

All patients underwent cardiac ultrasound and myocardial contrast echocardiography in the department of cardiology of the hospital on the 5th day after admission. The patient was placed in left decubitus position and underwent conventional ultrasound section, left ventricular long axis four-chamber view, three-chamber view and two-chamber view respectively for cardiac ultrasound examination. After routine cardiac ultrasound examination was completed, myocardial contrast echocardiography was performed. Shanghai Bolai sulfur six fluoride microbubble powder injection was used as contrast agent. High mechanical index $(\mathrm{Ml}=0.57)$ was used to release high energy pulses to destroy microbubbles, and then low mechanical index $(\mathrm{Ml}=0.07$ ) was used for contrast examination to observe the filling of contrast agent in each cardiac muscle segment. The following indexes were measured and recorded: left ventricular diastolic diameter (LVIDd), left ventricular end-systolic volume (LVESV), mitral e-peak velocity (MVE), mitral a-peak velocity (MVA), left ventricular mass index (LVMASSI), left ventricular ejection fraction (LVEF), and ventricular wall motion index (WMSI). WMSI was calculated as follows: According to the regulations of American Association of Cardiac Ultrasound[13], the left ventricular long axis fourchamber, three-chamber and two-chamber sections were divided into 16 segments. Myocardium of each segment was scored according to ventricular wall motion. Scoring criteria: 1 point: normal ventricular wall motion; 2 minutes: the motion of the chamber wall was weakened; 3 . The wall motion disappeared; 4 points: contradictory movement of chamber wall; $\mathrm{WMSI}=$ sum of scores of each segment/number of summary segments[14]. After contrast, score was given according to the filling degree of each myocardial 
contrast agent: 1: uniform and obvious perfusion of myocardial tissue; 2 points: mild uneven perfusion of myocardial tissue; 3 points: myocardial tissue perfusion defect; Contrast agent score index (CSI)= sum of fractions of each segment/total segment number[15].

\subsection{Statistical treatment}

SPSS 26.0 data software was used for statistical analysis. All measurement data were first tested for normality, and the data conforming to normal distribution was expressed as $(x \pm s)$. The comparison between the two groups was performed by $T$ test. Data that did not conform to the normal distribution was represented by $M(P 25, P 75)$, and non-parametric test was used for comparison between the two groups. Enumeration data were presented as an example (\%), and rank sum test was used for comparison between the two groups. Pearson or Spearman correlation analysis was selected according to whether the data type conforms to the normal distribution. Logistic regression analysis was used for multivariate analysis. ROC curve was used to calculate the prediction cut-off point of TyG index with the highest specificity and sensitivity. Bilateral test was adopted, and the test level was $P<0.05$.

\section{Results}

\subsection{Comparison of clinical and laboratory data of patients(Table 1)}

The preoperative D-dimer, proportion of Killip class at admission $₫ 1$,Peak CK-MB,neutrophil-lymphocyte ratio, triglyceride, fasting blood glucose and TyG index in the no reflow group were higher than those in the reflow group, and the systolic blood pressure at admission in the no-reflow group was lower than that in the reflow group, with statistical significance $(P<0.05)$. The rate of hospital death in patients without reflow was significantly higher than that of patients with normal reflow.

Table 1 Comparison of clinical and laboratory baseline characteristics between the no-reflux group and the reflux group 


\begin{tabular}{|c|c|c|c|}
\hline Variable & $\begin{array}{l}\text { no-reflow group } \\
n=309\end{array}$ & $\begin{array}{l}\text { reflow group } \\
n=728\end{array}$ & $P$ value \\
\hline Age,years & $66.15 \pm 4.84$ & $63.23 \pm 4.93$ & 0.106 \\
\hline Male & 156ه50.49囚 & $352 \varangle 48.35)$ & 0.830 \\
\hline $\mathrm{BMl}, \mathrm{Kg} / \mathrm{m}^{2}$ & $25.38 \pm 2.52$ & $25.16 \pm 2.23$ & 0.606 \\
\hline Hypertension & $160(51.78)$ & $419(57.56)$ & 0.618 \\
\hline Atrial fibrillation & $109(35.28)$ & $184(25.27)$ & 0.292 \\
\hline Smoker & $170(55.02)$ & $408(56.04 \rrbracket$ & 0.899 \\
\hline SBP at admission,mmHg & $116.60 \pm 34.85$ & $138.98 \pm 31.66$ & 0.003 \\
\hline DBP at admission,mmHg & $81.49 \pm 17.25$ & $83.37 \pm 16.76$ & 0.451 \\
\hline Heart rate at admission $\square b p m$ & $76.76 \pm 18.27$ & $81.82 \pm 20.03$ & 0.103 \\
\hline Killip class at admission 11 & $72(23.62)$ & $75(10.30)$ & 0.014 \\
\hline \multicolumn{4}{|l|}{ Echocardiography } \\
\hline Preoperative LVEF & $45.63 \pm 9.56$ & $48.34 \pm 9.13$ & 0.056 \\
\hline \multicolumn{4}{|l|}{ Preoperative use of medication } \\
\hline Aspirin & $24(7.77)$ & $85(11.68)$ & 0.679 \\
\hline$\beta$-blocker & $22(7.12)$ & $36(4.95)$ & 0.778 \\
\hline ACRI/ARB & $35(11.33)$ & $86(11.81)$ & 1.002 \\
\hline \multicolumn{4}{|l|}{ Biochemical indicators } \\
\hline Peak CK-MB,u/l & $2855.6 \pm 2246.2$ & $1660.9 \pm 1380.2$ & $\otimes 0.001$ \\
\hline Hypertroponin I(ng/ml) & $18220.30 \pm 21765.49$ & $13940.98 \pm 19973.21$ & 0.471 \\
\hline NT-proBNP,pg/ml & $3155.88 \pm 3860.49$ & $2369.55 \pm 4762.09$ & 0.287 \\
\hline Hs-Crp,mg/l & $24.68 \pm 32.30$ & $15.84 \pm 29.46$ & 0.191 \\
\hline D - dimer,mg/l & $2.26 \pm 4.91$ & $1.31 \pm 2.33$ & 0.027 \\
\hline White blood cells,10^9 & $10.78 \pm 3.69$ & $10.43 \pm 3.27$ & 0.495 \\
\hline Neutrophils $\left[10^{\wedge} 9\right.$ & $8.56 \pm 3.74$ & $7.73 \pm 3.08$ & 0.114 \\
\hline Monocytes,10^9 & $0.64 \pm 0.36$ & $0.62 \pm 0.32$ & 0.601 \\
\hline Lymphocyte,10^9 & $1.61 \pm 1.09$ & $1.93 \pm 1.41$ & 0.122 \\
\hline NLR & $7.88 \pm 6.23$ & $6.32 \pm 4.72$ & 0.033 \\
\hline
\end{tabular}




\begin{tabular}{|llll|}
\hline HDL-CImmol/I & $1.84 \pm 0.46$ & $1.88 \pm 0.43$ & 0.665 \\
\hline LDL-CImmol/I & $3.27 \pm 0.69$ & $3.01 \pm 0.71$ & 0.087 \\
\hline TCImmol/I & $4.61 \pm 1.28$ & $4.48 \pm 1.02$ & 0.513 \\
\hline FPG,mmol/I & $2.58 \pm 1.33$ & $1.45 \pm 0.94$ & 0.011 \\
\hline TyG index & $5.43 \pm 1.36$ & $4.97 \pm 0.56$ & 0.007 \\
\hline
\end{tabular}

2.2 Comparison of angiographic features in the reflow group with no-reflow group(Table 2)

Patients in the no-reflow group had longer operation time;higher proportion of aortic balloon counterpulsation;higher proportion of TIMI blood flow grade 0 and the rate of in hospital death than those in the reflow group.The difference was statistically significant (P区0.05).

Table 2 Comparison of angiographic features between non - reflux group and reflux group 


\begin{tabular}{|c|c|c|c|}
\hline Variable & $\begin{array}{l}\text { no-reflux group } \\
n=309\end{array}$ & $\begin{array}{l}\text { reflux group } \\
n=728\end{array}$ & $P$ value \\
\hline operation time(min) & $81.36 \pm 28.72$ & $67.70 \pm 22.60$ & 0.003 \\
\hline Number of stenosis vessels & & & 0.108 \\
\hline 1 & 63(20.39ه & 242(33.24) & \\
\hline 2 & 133(43.04) & $215(29.53)$ & \\
\hline 3 & 113(36.57) & 271(37.23) & \\
\hline \multicolumn{4}{|l|}{ Interventional vascular } \\
\hline right coronary artery & 109(35.28) & 232(31.87) & 0.731 \\
\hline anterior descending & $129(41.75)$ & $339(46.57)$ & 0.199 \\
\hline circumflex & $71(22.97)$ & 157(21.56) & 0.022 \\
\hline Preoperative TIMI blood flow & & & 0.007 \\
\hline 0 level & 245(79.29) & $426(58.52)$ & \\
\hline$\geq 1$ level & $64(20.71)$ & $302(41.48)$ & \\
\hline Thrombus suction & 123(39.81) & 223(30.63) & 0.066 \\
\hline Balloon dilation prior to stent placement & 109(35.28) & 184(25.27) & 0.287 \\
\hline Balloon dilated after stent placement & 139(44.98) & 271(37.23) & 0.318 \\
\hline reperfusion window & $6.27 \pm 2.50$ & $5.13 \pm 3.03$ & 0.078 \\
\hline Length of stent implantation[mm) & $24.02 \pm 6.86$ & $25.90 \pm 6.91$ & 0.115 \\
\hline Diameter of the implanted stent (mm) & $3.05 \pm 0.39$ & $3.02 \pm 0.40$ & 0.616 \\
\hline Use tirofiban & 158(51.13) & $455(62.50)$ & 0.209 \\
\hline Use Bivaludine & 182(58.90) & $345(47.39)$ & 0.208 \\
\hline Ventricular tachycardia/ventricular fibrillation & $16(5.18)$ & $36(4.95)$ & 0.311 \\
\hline $\begin{array}{l}\text { Blood pressure decreased } \\
\text { during the operation }\end{array}$ & $38(12.30)$ & 61(8.38) & 0.392 \\
\hline Intraaortic Balloon Pump & $29(9.40)$ & 13(1.79) & 0.003 \\
\hline Pacemaker placement & $47(15.21)$ & $49(6.73)$ & 0.166 \\
\hline In hospital death & $25(8.1)$ & 19(2.6) & $\varangle 0.001$ \\
\hline
\end{tabular}


2.3 Comparison of echocardiography and myocardial contrast echocardiography between the no-reflow group and the reflow group(Table 3)

The indexes of cardiac ultrasound and myocardial contrast echocardiography on the 5th day after admission of patients in the no-reflow group and the reflow group suggested that the Left ventricular endsystolic volume(LVESV), Ventricular wall motion score index(WMSI) and Contrast agent score index(CSI) of patients in the no-reflow group were higher than those in the reflow group, while the Left ventricular ejection fraction(LVEF) was lower than those in the reflow group, with statistically significant differences(Pख0.05).

Table3 Comparison of echocardiography and myocardial contrast echocardiography between the non reflow group and the reflow group

\begin{tabular}{|clll|}
\hline Variable & $\begin{array}{l}\text { no-reflux group } \\
\mathrm{n}=309\end{array}$ & $\begin{array}{l}\text { reflux group } \\
\mathrm{n}=728\end{array}$ & P value \\
\hline LVEDV,ml & $128.26 \pm 31.13$ & $125.27 \pm 25.04$ & 0.312 \\
\hline LVESV,ml & $62.78 \pm 20.98$ & $49.83 \pm 14.56$ & 0.023 \\
\hline LVEF,\% & $51.34 \pm 8.03$ & $56.93 \pm 8.75$ & 0.039 \\
\hline LVIDd,mm & $53.06 \pm 6.23$ & $48.93 \pm 6.16$ & 0.357 \\
\hline MVE,m/s & $0.65 \pm 0.11$ & $0.60 \pm 0.14$ & 0.633 \\
\hline MVA,m/s & $0.75 \pm 0.16$ & $0.71 \pm 0.13$ & 0.599 \\
\hline LVMASSI,g/m ${ }^{2}$ & $106.74 \pm 24.28$ & $96.45 \pm 23.47$ & 0.108 \\
\hline WMSI & $1.46 \pm 0.19$ & $1.18 \pm 0.14$ & 0.016 \\
\hline CSI & $1.82 \pm 0.33$ & $1.35 \pm 0.15$ & 0.011 \\
\hline
\end{tabular}

\subsection{Logistic regression analysis of risk factors with no reflow}

In the multivariate regression analysis, variables such as duration of surgery and aortic balloon counterpulsation were excluded, which were believed to be the result of the absence of reflow after PCI. Since the calculation of TyG index involved fasting blood glucose and triglyceride, the risk factors mentioned above were excluded in the multivariate analysis. Multivariate regression analysis showed that TyG index;Ventricular wall motion score index(WMSI) and Contrast agent score index(CSI) were independent risk factors for no reflow after $\mathrm{PCl}$, and the difference was statistically significant $(\mathrm{P}<0.05)$. (Table 4)

Table 4 Risk factors of no-reflow analyzed by Logistic regression analysis 


\begin{tabular}{|c|c|c|c|c|c|c|}
\hline \multirow[t]{2}{*}{ Variable } & \multicolumn{3}{|c|}{ Monofactor analysis } & \multicolumn{3}{|c|}{ Multivariate analysis } \\
\hline & OR & $95 \% \mathrm{Cl}$ & $\begin{array}{l}P \\
\text { Value }\end{array}$ & OR & $95 \% \mathrm{Cl}$ & $\begin{array}{l}P \\
\text { Value }\end{array}$ \\
\hline Tyg index & 1.590 & $\begin{array}{l}1.313- \\
1.925\end{array}$ & $\triangle 0.001$ & 1.484 & $\begin{array}{l}1.203- \\
1.831\end{array}$ & $\varangle 0.001$ \\
\hline D - dimer & 2.520 & $\begin{array}{l}1.289- \\
4.924\end{array}$ & 0.009 & 2.556 & $\begin{array}{l}1.220- \\
5.351\end{array}$ & 0.113 \\
\hline NLR & 1.056 & $\begin{array}{l}1.000- \\
1.116\end{array}$ & 0.037 & 1.030 & $\begin{array}{l}0.970- \\
1.094\end{array}$ & 0.290 \\
\hline $\begin{array}{l}\text { Killip classificationप1 at } \\
\text { admission }\end{array}$ & 2.544 & $\begin{array}{l}1.183- \\
5.466\end{array}$ & 0.014 & 1.625 & $\begin{array}{l}0.504- \\
3.168\end{array}$ & 0.599 \\
\hline LVEF & 0.033 & $\begin{array}{l}0.921- \\
1.383\end{array}$ & 0.018 & 1.534 & $\begin{array}{l}0.754- \\
3.122\end{array}$ & 0.240 \\
\hline LVESV & 1.311 & $\begin{array}{l}0.684- \\
2.513\end{array}$ & 0.420 & 1.192 & $\begin{array}{l}0.531- \\
2.678\end{array}$ & 0.676 \\
\hline WMSI & 3.603 & $\begin{array}{l}1.532- \\
8.515\end{array}$ & 0.006 & 2.640 & $\begin{array}{l}1.036- \\
4.722\end{array}$ & 0.039 \\
\hline CSI & 2.589 & $\begin{array}{l}1.343- \\
4,994\end{array}$ & 0.007 & 2.299 & $\begin{array}{l}1.117- \\
4.767\end{array}$ & 0.022 \\
\hline
\end{tabular}

\subsection{ROC Curve Analysis}

The area under the ROC curve predicted by TyG index after $\mathrm{PCl}$ without reflow was $0.809,95 \% \mathrm{Cl}(0.740$ 0.879), higher than CSI and WMSI.When the TyG index was 6.995, the sensitivity and specificity of Youden index were $82.4 \%$ and $70.3 \%$ respectively at the maximum. The area under the curve (AUC) of CSI and WMSI are respectively $0.77995 \% \mathrm{Cl}(0.713-0.846)$ and $0.65795 \% \mathrm{Cl}(0.530-0.693)$.

\section{Discussion}

In this study, we found that TyG index could be used as an independent predictor of no reflow phenomenon in actue myocardial infarction patients after PCl. To our knowledge, this is the first study to evaluate the relationship between TyG index and the absence of reflow after $\mathrm{PCl}$.

With an increasing number of deaths due to acute myocardial infarction each year, $\mathrm{PCl}$ has become a common surgical treatment for acute myocardial infarction in addition to conventional drug therapy. It can open blood vessel in time, save ischemic myocardium.After PCl, there was still abnormal perfusion at the myocardial level. This phenomenon of "no reflow" was attributed to abnormal myocardial microcirculation, resulting in serious damage of myocardial cells, has been proved to be associated with short-term and long-term prognosis of patients[16]. And so it proved in our study,the rate of hospital death in patients without reflow was significantly higher than that of patients with normal reflow. 
Currently, the indexes commonly used in clinical evaluation of perfusion level include myocardial perfusion color grading;cardiac PET-CT; myocardial contrast echocardiography and TIMI blood flow grading [17-18], both of which are invasive and expensive. Therefore, a simple index that can be used to evaluate the myocardial perfusion level of patients after $\mathrm{PCl}$ is needed.

At present, studies have suggested that the occurrence of "no reflow"phenomenon may be related to factors such as inflammatory state[19], coronary artery microcirculation disorder[20], vasospasm[21], thrombosis [22]and so on. In our study, it was found that the D-dimer, Neutrophil-lymphocyte ratio, triglyceride and fasting blood glucose of patients in the no reflow group were all higher than those in the reflow group;which confirmed the effect of the above factors and was consistent with the results of previous studies[23-25].LVESV,WMSI,CSI also higher than the reflow group;It is suggested that the patients with no reflow have poor blood supply and weak cardiac activity[26].

Fasting glucose is mainly used to reflect the secretion of insulin in the body. When the sugar content in blood and tissues in the body increases, the body is prone to sugar metabolism disorder. Hyperglycemia, on the one hand, it accelerates the injury of endothelial cells in the body[27]; On the other hand, high fasting glucose level is also easy to promote the body thrombosis[28]. Triglycerides are mainly found in very low density lipoprotein cholesterol and are easily converted to low density lipoprotein cholesterol in the plasma. Low density lipoprotein cholesterol (LDL-C) is rapidly glycosylated to form AGE-LDL complex under the condition of high blood sugar. AGE-LDL has a strong atherogenic effect, which has been proved to be related to the absence of reflow in patients after PCI[29]. TyG index combines two risk factors, fasting blood glucose and triglyceride, to form a novel composite index. It has been shown to be associated with hypertension[30], atherosclerosis[31], coronary heart disease[32], and myocardial infarction[33], and poor prognosis for patients[34]. Based on the above research results, we speculated that TyG index was related to the absence of reflow after PCl. At the same time, this hypothesis has been confirmed in our study. Logistic multivariate analysis showed that TyG index could be used as an independent risk factor for the occurrence of no reflow phenomenon in STEMI patients after PCI.The area under the curve is larger than traditional indicators:CSI and WMSI.Compared with CSI and WMSI, TyG index can better predict the occurrence of non-reflow after PCl.

At present, the mechanism of TyG index and no-flow phenomenon is not clear. According to the existing research results, it is speculated that the high insulin resistance level in the body may be related to promoting the secretion of inflammatory mediators, accelerating thrombosis and reducing collateral circulation[35]. In patients with high insulin resistance, fibrinolytic ability of the body is reduced, which leads to platelet aggregation, and endogenous anti-fibrinolytic substances (PAl-1), fibrinogen, von willebrand factor (VWF), $\mathrm{X}$ factor and other coagulant substances are significantly increased[36]. All these factors can lead to myocardial microcirculation disorder and no reflow phenomenon.

This study has the following limitations: It is a single center retrospective study with a small sample size, and there is selection bias. In addition, only acute myocardial infarction patients were included in this study,the results of the study can not be generalized to other populations and patients undergoing 
PCl.Lastly,Limited by the small sample size and event rate, the prognostic value of TyG index in acute myocardial infarction patients after $\mathrm{PCl}$ was not further evaluated.

\section{Conclusion}

TyG index has important predictive value in acute myocardial infarction patients with no reflow after $\mathrm{PCl}$.It is used for risk stratification of patients at high risk of no reflow before PCI.Timely preventive measures were taken to reduce the occurrence of no reflow after operation.

\section{Declarations}

Funding The authors received the fifth batch of medical key discipline projects in Jiading District, Shanghai(2020-jdyxzdxk-16) support for the research.

\section{Compliance with ethical standards}

Conflicts of interest: All authors declare that they have no conflict of interest.

\section{References}

1. Neri M, Fineschi V, Di Paolo $M$, et al. Cardiac oxidative stress and inflammatory cytokines response after myocardial infarction. Curr Vasc Pharmacol. 2015;13(1):26-36.

2. Chan W, Stub D, Clark DJ, Ajani AE, Andrianopoulos N, Brennan AL, New G, Black A, Shaw JA, Reid $\mathrm{CM}$, Dart AM and Duffy SJ. Usefulness of transient and persistent no reflow to predict adverse clinical outcomes following percutaneous coronary intervention. The American journal of cardiology. 2012;109:478-85.

3. Porter TR, Mulvagh SL, Abdelmoneim SS, et al. Clinical Applications of Ultrasonic Enhancing Agents in Echocardiography: 2018 American Society of Echocardiography Guidelines Update. J Am Soc Echocardiogr. 2018;31(3):241-274.

4. Valero SJ, Moreno R, Reyes RM, et al. Pharmacological approach of no-reflow phenomenon related with percutaneous coronary interventions. Cardiovasc Hematol Agents Med Chem. 2008;6(2):125129.

5. Nishio M, Ueda Y, Matsuo K, et al. Association of target lesion characteristics evaluated by coronary computed tomography angiography and plaque debris distal embolization during percutaneous coronary intervention. Circ J. 2014;78(9):2203-2208.

6. Tartan Z, Ozer N, Uyarel H, et al. Metabolic syndrome is a predictor for an ECG sign of no-reflow after primary PCl in patients with acute ST-elevation myocardial infarction. Nutr Metab Cardiovasc Dis. 2008;18(6):441-447.

7. Gesteiro E, Bastida S, Barrios L, Sánchez-Muniz FJ. The triglyceride-glucose index, an insulin resistance marker in newborns?. Eur J Pediatr. 2018;177(4):513-520. 
8. Won KB, Park EJ, Han D, et al. Triglyceride glucose index is an independent predictor for the progression of coronary artery calcification in the absence of heavy coronary artery calcification at baseline. Cardiovasc Diabetol. 2020;19(1):34. Published 2020 Mar 16.

9. Zhang S, Du T, Zhang J, et al. The triglyceride and glucose index (TyG) is an effective biomarker to identify nonalcoholic fatty liver disease. Lipids Health Dis. 2017;16(1):15. Published 2017 Jan 19.

10. Li X, Li G, Cheng T, Liu J, Song G, Ma H. Association between triglyceride-glucose index and risk of incident diabetes: a secondary analysis based on a Chinese cohort study : TyG index and incident diabetes [published correction appears in Lipids Health Dis. 2021 Jan 30;20(1):8]. Lipids Health Dis. 2020;19(1):236. Published 2020 Nov 8.

11. Zhao S, Yu S, Chi C, et al. Association between macro- and microvascular damage and the triglyceride glucose index in community-dwelling elderly individuals: the Northern Shanghai Study. Cardiovasc Diabetol. 2019;18(1):95. Published 2019 Jul 25.

12. Vafaie M. State-of-the-art diagnosis of myocardial infarction. Diagnosis (Berl). 2016;3(4):137-142.

13. Schiller NB, Shah PM, Crawford M, et al. Recommendations for quantitation of the left ventricle by two-dimensional echocardiography. American Society of Echocardiography Committee on Standards, Subcommittee on Quantitation of Two-Dimensional Echocardiograms. J Am Soc Echocardiogr. 1989;2(5):358-367.

14. Galiuto L, Gabrielli FA, Lanza GA, et al. Influence of left ventricular hypertrophy on microvascular dysfunction and left ventricular remodelling after acute myocardial infarction. Eur $\mathrm{J}$ Echocardiogr. 2010;11(8):677-682.

15. Olszowska M, Kostkiewicz M, Podolec P, Rubis P, Tracz W. Myocardial viability detected by myocardial contrast echocardiography--prognostic value in patients after myocardial infarction. Echocardiography. 2010;27(4):430-434.

16. Papapostolou S, Andrianopoulos N, Duffy SJ, et al. Long-term clinical outcomes of transient and persistent no-reflow following percutaneous coronary intervention (PCI): a multicentre Australian registry. Eurolntervention. 2018;14(2):185-193.

17. Danijela T, Jelena D, Olga P, Zorana VP. Assessment of Coronary Microcirculation with Myocardial Contrast Echocardiography. Curr Pharm Des. 2018;24(25):2943-2949.

18. Sun $L$, Wang $Z$, Xu T, et al. The value of real-time myocardial contrast echocardiography for detecting coronary microcirculation function in coronary artery disease patients. Anatol $\mathrm{J}$ Cardiol. 2018;19(1):27-33.

19. Niccoli G, Lanza GA, Spaziani C, et al. Baseline systemic inflammatory status and no-reflow phenomenon after percutaneous coronary angioplasty for acute myocardial infarction. Int $\mathrm{J}$ Cardiol. 2007;117(3):306-311.

20. Feher A, Chen SY, Bagi Z, Arora V. Prevention and treatment of no-reflow phenomenon by targeting the coronary microcirculation. Rev Cardiovasc Med. 2014;15(1):38-51.

21. Niccoli G, Lanza GA, Spaziani C, et al. Baseline systemic inflammatory status and no-reflow phenomenon after percutaneous coronary angioplasty for acute myocardial infarction. Int J Cardiol. 
2007;117(3):306-311.

22. Feher A, Chen SY, Bagi Z, Arora V. Prevention and treatment of no-reflow phenomenon by targeting the coronary microcirculation. Rev Cardiovasc Med. 2014;15(1):38-51.

23. Simsek B, Cinar T,Ozan V, et al. The association of acute--to--chronic glycemic ratio with no-reflow in patients with ST-segment elevation myocardial infarction undergoing primary percutaneous coronary intervention.Kardiol Pol. 2021;79(2):170-178.

24. .Li H, Fu DG, Liu FY, Zhou H, Li XM. Evaluation of related factors, prediction and treatment drugs of no-reflow phenomenon in patients with acute ST-segment elevation myocardial infarction after direct PCl. Exp Ther Med. 2018;15(4):3940-3946.

25. Turkmen S, Dogdu O, Tekin K, et al. The relationship between neutrophil/lymphocyte ratio and the TIMI flow grade in patients with STEMI undergoing primary PCI. Eur Rev Med Pharmacol Sci.2013;17(16):2185-2189.

26. ,Abdelmoneim SS, Martinez MW, Mankad SV, et al. Resting qualitative and quantitative myocardial contrast echocardiography to predict cardiac events in patients with acute myocardial infarction and percutaneous revascularization. Heart Vessels. 2015;30(1):45-55.

27. Fiorentino TV, Prioletta A, Zuo P, Folli F. Hyperglycemia-induced oxidative stress and its role in diabetes mellitus related cardiovascular diseases. Curr Pharm Des. 2013;19(32):5695-5703.

28. Zuurbier SM, Hiltunen S, Tatlisumak T, et al. Admission Hyperglycemia and Clinical Outcome in Cerebral Venous Thrombosis. Stroke. 2016;47(2):390-396.

29. Zhu L, He Z, Wu F, et al. Immunization with advanced glycation end products modified low density lipoprotein inhibits atherosclerosis progression in diabetic apoE and LDLR null mice. Cardiovasc Diabetol. 2014;13:151. Published 2014 Nov 13.

30. Zheng R, Mao Y. Triglyceride and glucose (TyG) index as a predictor of incident hypertension: a 9year longitudinal population-based study. Lipids Health Dis. 2017;16(1):175. Published 2017 Sep 13.

31. Chiu TH, Tsai HJ, Chiou HC, Wu PY, Huang JC, Chen SC. A high triglyceride-glucose index is associated with left ventricular dysfunction and atherosclerosis. Int J Med Sci. 2021;18(4):10511057. Published 2021 Jan 1.

32. da Silva A, Caldas APS, Hermsdorff HHM, et al. Triglyceride-glucose index is associated with symptomatic coronary artery disease in patients in secondary care. Cardiovasc Diabetol. 2019;18(1):89. Published 2019 Jul 11.

33. Liu Y, Wu M, Xu J, Sha D, Xu B, Kang L. Association between Triglyceride and glycose (TyG) index and subclinical myocardial injury. Nutr Metab Cardiovasc Dis. 2020;30(11):2072-2076.

34. Luo E, Wang D, Yan G, et al. High triglyceride-glucose index is associated with poor prognosis in patients with acute ST-elevation myocardial infarction after percutaneous coronary intervention. Cardiovasc Diabetol. 2019;18(1):150. Published 2019 Nov 13.

35. Tartan Z, Ozer N, Uyarel $\mathrm{H}$, et al. Metabolic syndrome is a predictor for an ECG sign of no-reflow after primary PCl in patients with acute ST-elevation myocardial infarction. Nutr Metab Cardiovasc Dis. 2008;18(6):441-447. 
36. Devaraj S, Xu DY, Jialal I. C-reactive protein increases plasminogen activator inhibitor-1 expression and activity in human aortic endothelial cells: implications for the metabolic syndrome and atherothrombosis. Circulation. 2003;107(3):398-404.

\section{Figures}

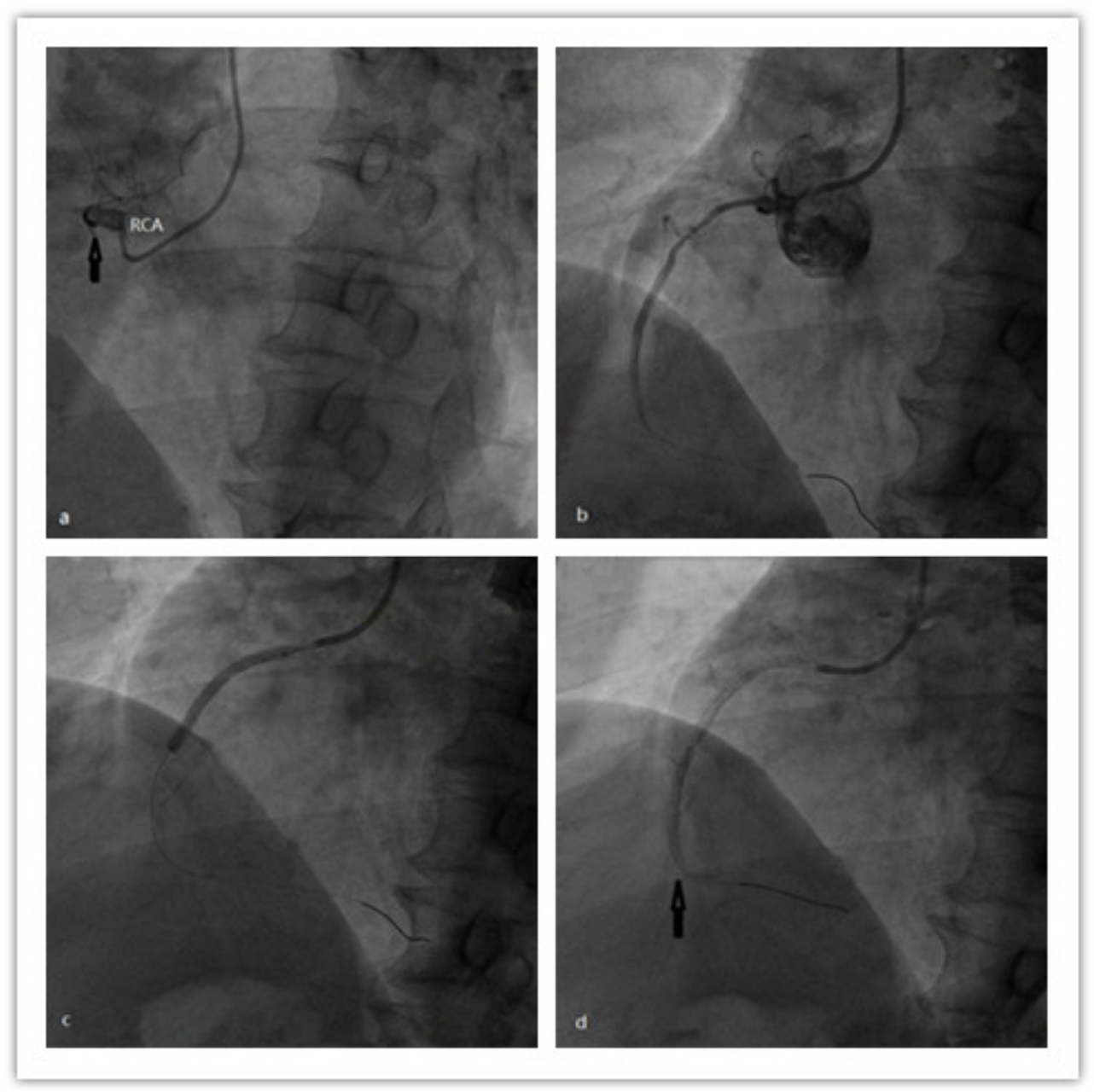

\section{Figure 1}

A 55-year-old male was diagnosed with acute inferior wall myocardial infarction. His TyG index was calculated at 7.28.a. The RCA was completely occluded below the proximal segment, and the anterior flow was TIMI0.b.Thrombusterll thrombus aspiration catheter was administered to coronary thrombus aspiration and reexamination and angiography.c.rapamycin stent (Excel $3.5 * 36 \mathrm{~mm} 14 \mathrm{ATM}$ ) was implanted at the RCA d.Re-examination and angiography showed that there was no reflow below the middle segment of RCA.(TIMI flow 1) TyG index serum triglyceride and fasting glycemic index;RCA Right coronary artery $\mathbb{T} \mathrm{TIMI}$ thrombolysis in myocardial infarction. 


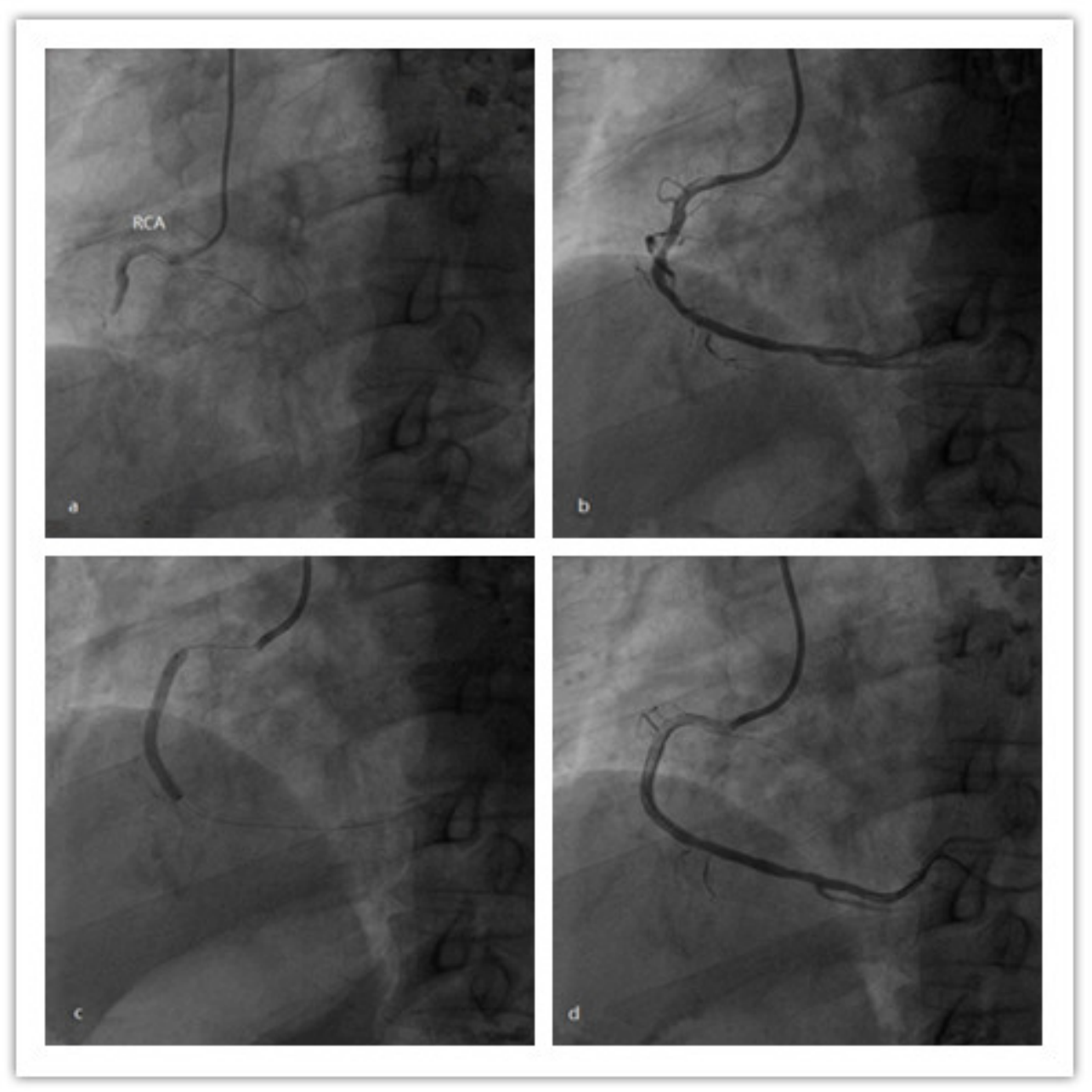

Figure 2

A 58-year-old male was diagnosed with acute inferior wall myocardial infarction. His TyG index was calculated at 6.44.a.RCA was completely occluded below the proximal middle segment, with local thrombotic shadow and TIMI0 grade of forward flow.b.2.0* $15 \mathrm{~mm}$ balloon predilation of RCA near midcourse lesions.c.Implantation of rapamycin drug stent (Partner $3.5^{\star} 36 \mathrm{~mm}$ ) for RCA near midcourse lesions.d.TIMI 3 flow was obtained at RCA artery. TyG index serum triglyceride and fasting glycemic index;RCA Right coronary artery $₫ \mathrm{TIMI}$ thrombolysis in myocardial infarction. 


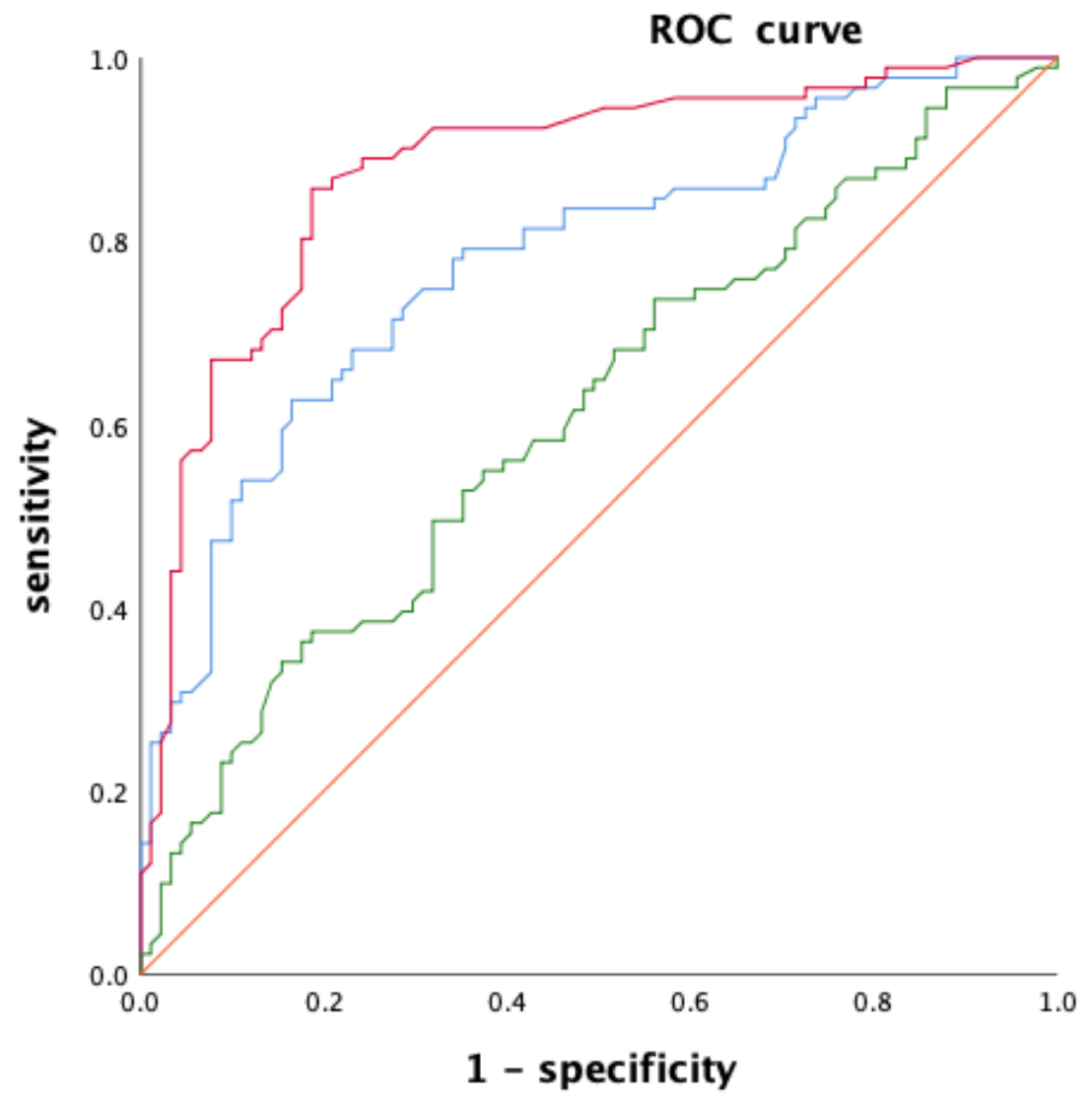

Source of the Curve CSI TyG INDEX WMSI

Reference

Figure 3

Legend not included with this version 